\title{
Facial Scars Reconstruction
}

\section{Luc Téot}

\section{Contents}

38.1 Objectives of the Chapter - 326

38.2 Techniques $-\mathbf{3 2 6}$

38.3 Critical Analysis of the Literature $\mathbf{- 3 3 0}$

References - 331 


\subsection{Objectives of the Chapter}

The objective of the chapter is to present the different reconstruction techniques, distinctly describing the ones recruiting autologous skin and subcutaneous tissues, transferred from surrounding color-matching donor site areas as well as techniques suitable for a complete face transplantation from a living human donor, with this homologous transfer being realized using microsurgical revascularization.

The aim is to minimize as far as possible the consequences of facial scars, which induce a loss of the dynamic aspect of the face and loss of the specific mimics of each human character to express different emotions. Consequences of the different surgical strategies are described, including local, regional, and systemic potential complications.

\subsection{Techniques}

\section{Skin Resurfacing}

Used in postburns scars involving retractions and loss of skin capacity, the resurfacing using dermal substitute and partial thickness skin grafts is the easiest technique to perform. Excision of the scars is done via a long meticulous surgical debridement, preventing to severe venous and arterial pedicles, resecting the hard thick scar tissue, which may be strongly adherent to the muscular and aponeurotic structures of the face around the orifices, mouth, nose, and ears. The neck is extensively freed from retracting scars, allowing a restauration of the movements up and down and laterally on each side. Covering using dermal substitute has become more and more popular in burns surgery since the initial works on Integra [1].

\section{Pedicled-Expanded Deltopectoral Flaps}

Among the commonly used donor sites, the deltopectoral site was conceived as one of the most suitable donor tissues for the reconstruction in the face and neck for its adjacent site and match in color and texture [2]. This technique uses an island flap, which is prepared and expanded to the size of the surface to be covered over the face and the distance of the flap pedicle to the distal end of the flap. This is a two-staged procedure. The size and projection of the silicone expander should precisely be determined before the first surgical step. Thanks to a limited incision outside the future face flap which will be drawn over the dorsal skin, a large expander will be inserted in a cavity created subcutaneously between the subcutaneous fat and the underlying muscle aponeurosis. The expander is chosen based on its capacity of inducing a predetermined skin projection, surface of the projected area, and length and width. The expander is connected via a silicone tubing to a valve which is also inserted under the skin at a limited distance from the expander edges. After a period of 2 weeks, the expander filling will start, and the amount of liquid determined on the rate of filling and the needed projection to obtain the desired skin expansion. Usually the skin expander is overexpanded to 1.5 of its volume, in order to obtain an even skin expansion. Possible complications may include extravasation of the expander (in case of rigid silicone angle), a too rapid expansion inducing a local devascularization and stretch marks, the long distance from the back to the face and the postop edema sometimes causing flap tip necrosis

But a good skin compliance, normal contours, and emotional expression are usually noted after the reconstruction.

3. Prefabrication

In 2000, the main author proposed the neofabrication of a cervico-deltopectoral flap used as an island flap after skin perfusion boosting. This technique, called prefabrication, was developed on the principle that when a vascular carrier (a fascia including an artery and a vein) is inserted between the expanded skin and the silicone balloon, the expanded skin territory can be extended and its neovascularization amplified. The technique was published in 2000 in The Lancet, opening the way to the creation of flaps larger than the normal anatomy allows it [3].

Technically, the radial forearm facial flap $(20 \mathrm{~cm} \times 6 \mathrm{~cm})$ is harvested with the radial artery and the vein, which are microsurgically branched on the facial vessels artery and vein using a limited incision. The fascia is inserted subcutaneously over the clavicular bone anteriorly, the trapeze muscles posteriorly, and the deltoid muscle laterally. A transfixient suture is needed at the distal end of the fascia in order to prevent any gliding possibility.

Depending on the surface to cover, the amount of expansion and size and capacity of the expander may vary. When the complete face should be covered, a 2-liter skin expander will be inserted between the vascularized fascia and the underlying structures. The valve and tubing are positioned posteriorly on the back.

A progressive filling of the skin expander is realized twice a week, with a regimen allowing to get the surface of the face $(25 \mathrm{~cm} \times 30 \mathrm{~cm})$ on the shoulder after 3-4 months. An arteriogram is realized before the second stage in order to confirm the viability of the fascia and its capacity to revascularize the extra skin obtained thanks to the skin expansion. This arteriogram shows all the new arterial ramifications emerging from the radial transplanted artery, a sign which was interpreted by the radiologists as a certi- 

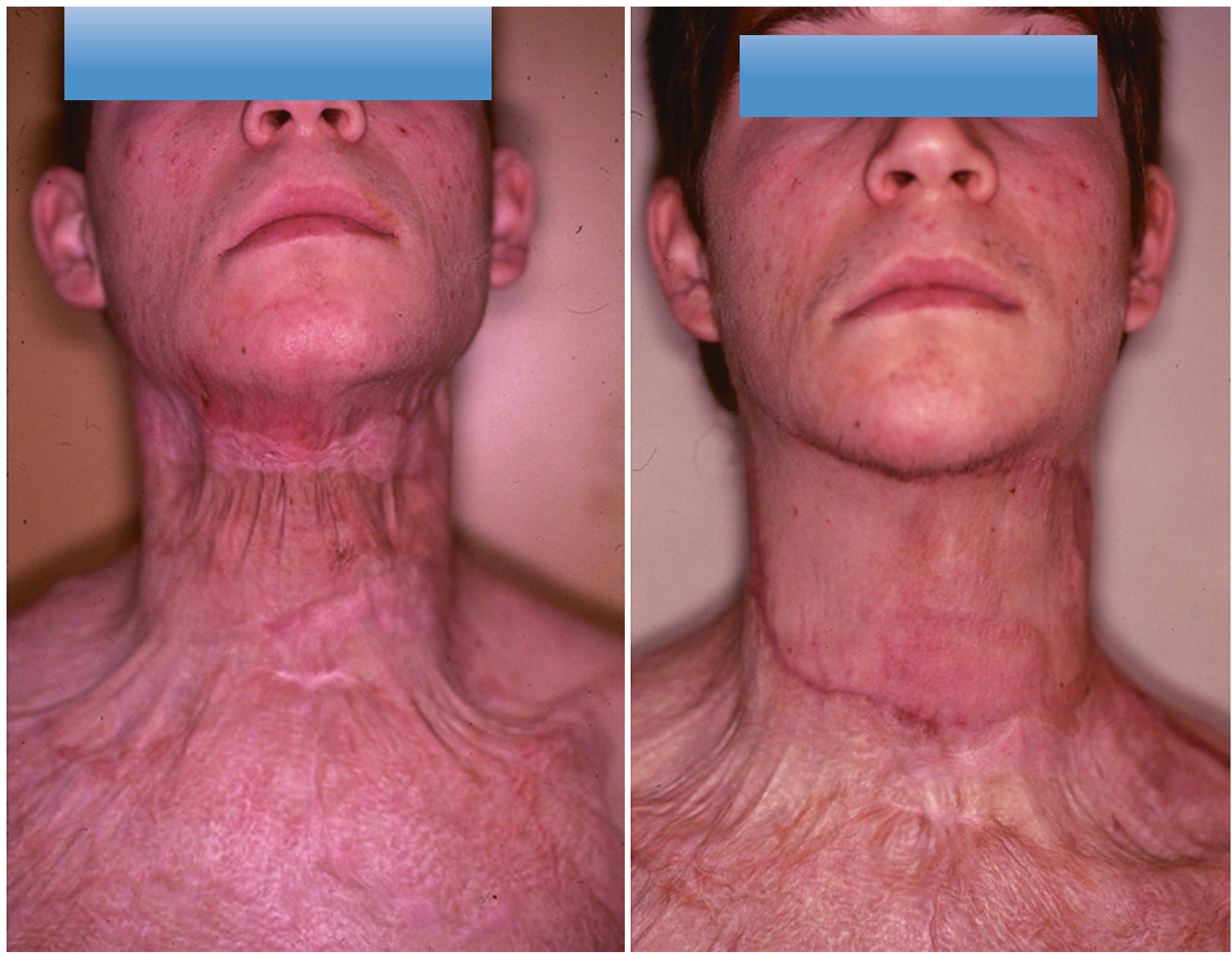

- Fig. 38.1 Before and after prefabricated flap on the neck of a young male presenting a contractile postburn scar

tude that the fascial vessels were inducing the new flap vascularization.

During the second step, a flap large enough to cover the entire face (if needed) is designed over the expanded skin. The dissection starts from lateral to medial over the shoulder, with a particular attention not to harm the vascular pedicle. Once the flap is harvested and the flap pedicled on the arteriovenous pedicle, all facial scars are removed deep to the fascia, in order to get a maximum surface covered by the new flap. Orifices (mouth, eyes, nostrils) are opened in front of the anatomical corresponding zones.

Long-term results are good, with the transplanted skin depth remaining adapted to the volume of the face, thanks to the precompression realized during the skin expansion (- Figs. 38.1, 38.2, 38.3, 38.4, 38.5, 38.6, and 38.7).

This technique allows to get a precompressed large piece of skin, well vascularized and conformable. The whole face can be covered with a large viable piece of skin. There is no need for complementary drugs.

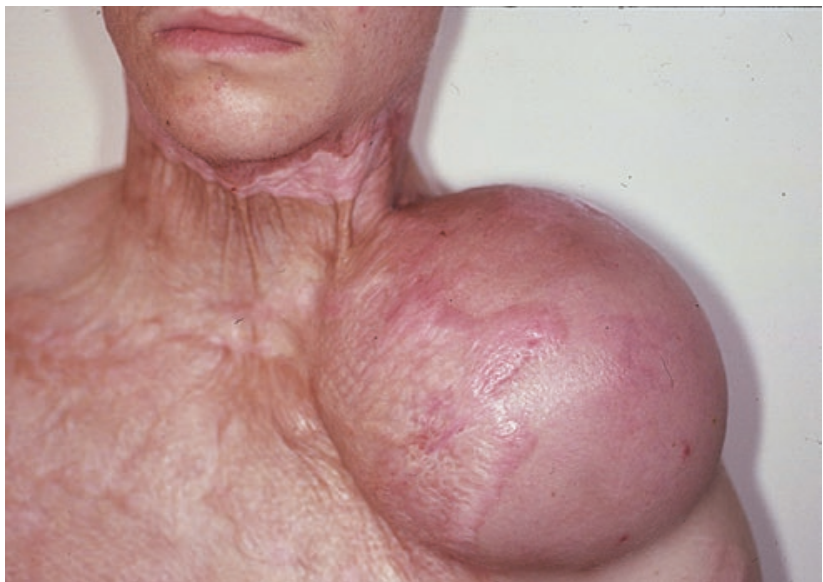

- Fig. 38.2 Aspect of the expanded skin flap before the second procedure

\section{Superthin Flaps}

The "superthin flap" concept was introduced by Ogawa et al. in 1994 [4], based on the subdermal vascular network (SVN). 


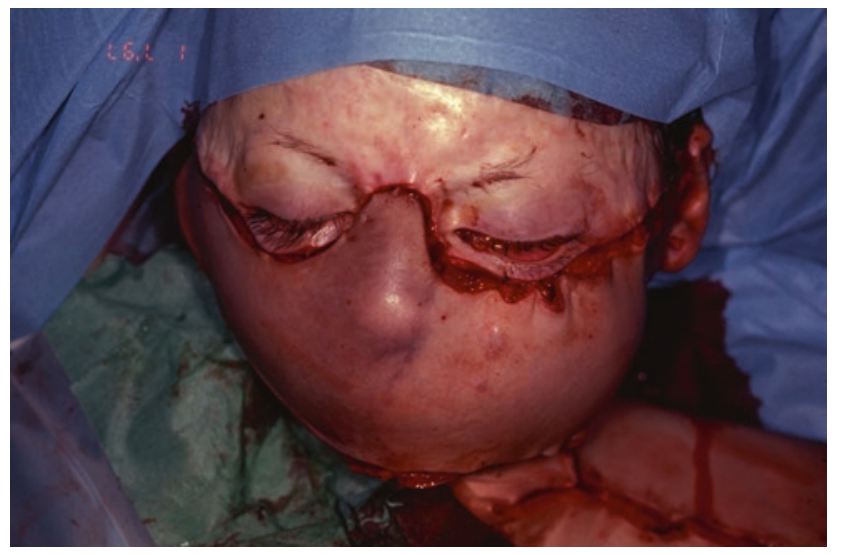

- Fig. 38.3 During the second procedure, the orifices (mouth, nose,) must be reopened adequately

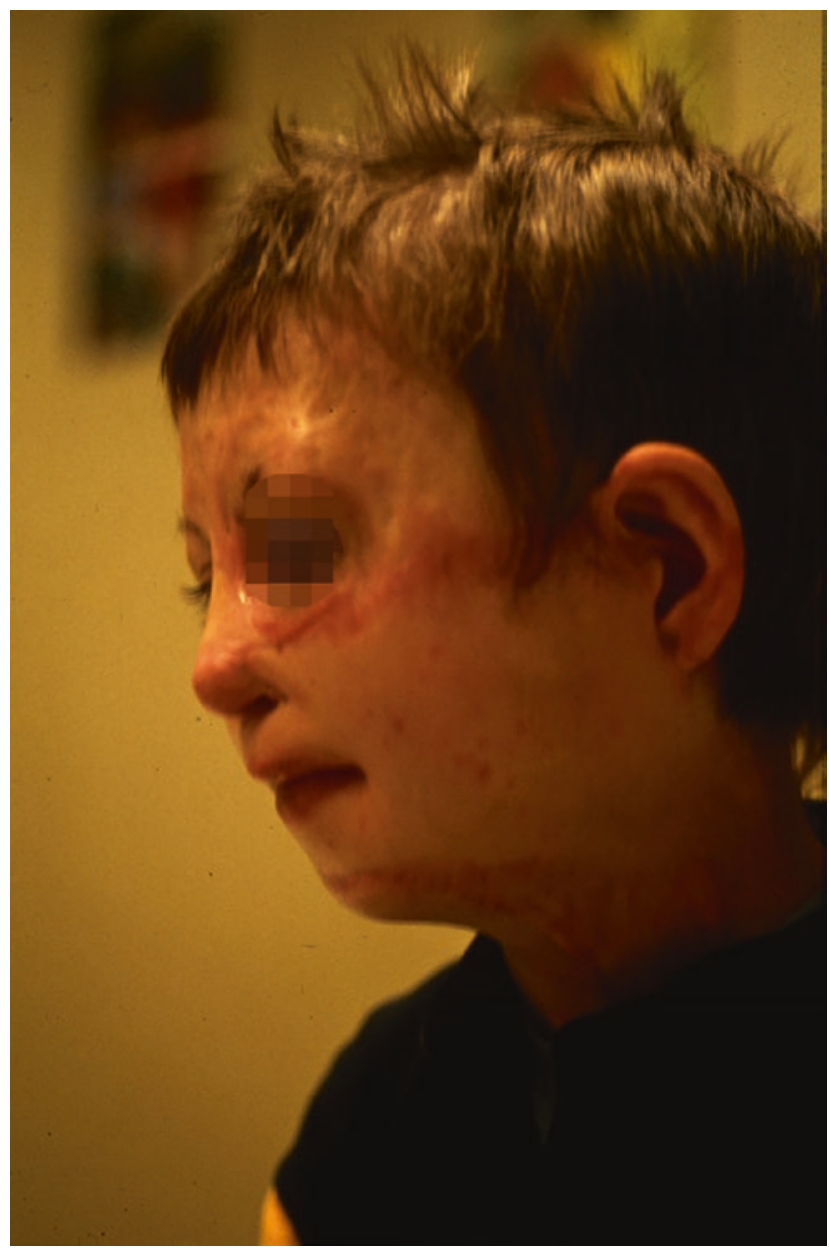

Fig. 38.4 One year postoperative aspect

Superthin flaps were used in different clinical situations. Twenty-one expanded flaps were used to reconstruct 21 face or neck scar cases in 9 males and 12 females. In the first operation, an expander was inserted on the fascia of the pectoralis major muscle, and then about $1000 \mathrm{cc}$ of saline was injected during a 2-month period. In the second operation, the flap was thinned primarily and applied to the recipient site. Three weeks after the second operation, the pedicle of the flap was cut down and sutured.

In 2007, the authors reported an expanded "superthin flap" for reconstruction of the face and neck for the first time in a patient.

In their series the authors reported different flap sizes, ranging from $4 \mathrm{~cm} \times 14 \mathrm{~cm}$ to $10 \mathrm{~cm} \times 22 \mathrm{~cm}$. Expanded volume ranged from $800 \mathrm{cc}$ to $1200 \mathrm{cc}$. All flaps survived completely, and scar tissues were replaced with normal skin. Flaps did not shrink after the operations, and contractures did not recur. The main advantages of the expanded flaps are the potential to create large thin flaps with a texture and color matching with the recipient area. The donor site can be closed primarily; and microsurgery is not required. However, the disadvantage of the method is the requirement for two or three operations.

The advantages and limits of the techniques are using skin expansion with or without microsurgery. This technique may be considered safe in terms of skin manipulations, the obtained tissue being conformable enough to redrape the whole face without lymphatic accumulation. Edema can be maintained at a low level, especially when using complementary compressive techniques comparable to the ones used in postburn-grafted faces. However, the second stage (flap positioning) after skin expansion is crucial in order to prevent distal regions of the flap to be partially devascularized and become distally necrosed, a complication issuing to a potential tissue loss. The transferred tissues are coming from areas where the dermal component is thicker than desired for facial tissues like the eyebrows or lips, but the global aspect of the transplanted face is compatible with a normal life, thanks to a daily adapted makeup and some retouching on the eyebrows and the lips. The skin has been submitted during skin expansion to a pressure which limits the depth and gives a thin texture. However, in most of the cases, complementary surgical retouches will be needed for refining the red lips, nasal skin depth and nostrils, columella, and eyebrows, reshaped using liposuction, fat transfer, and mucosal surgery

5. Facial Allotransplantations

Historical Aspects

The Pioneers

The first clinical case was realized in November 27, 2005, in Amiens (France); the patient presented a disfiguration after a severe dog bite. She was submitted to the world's first partial face transplant This disruptive approach established loss of quality of 

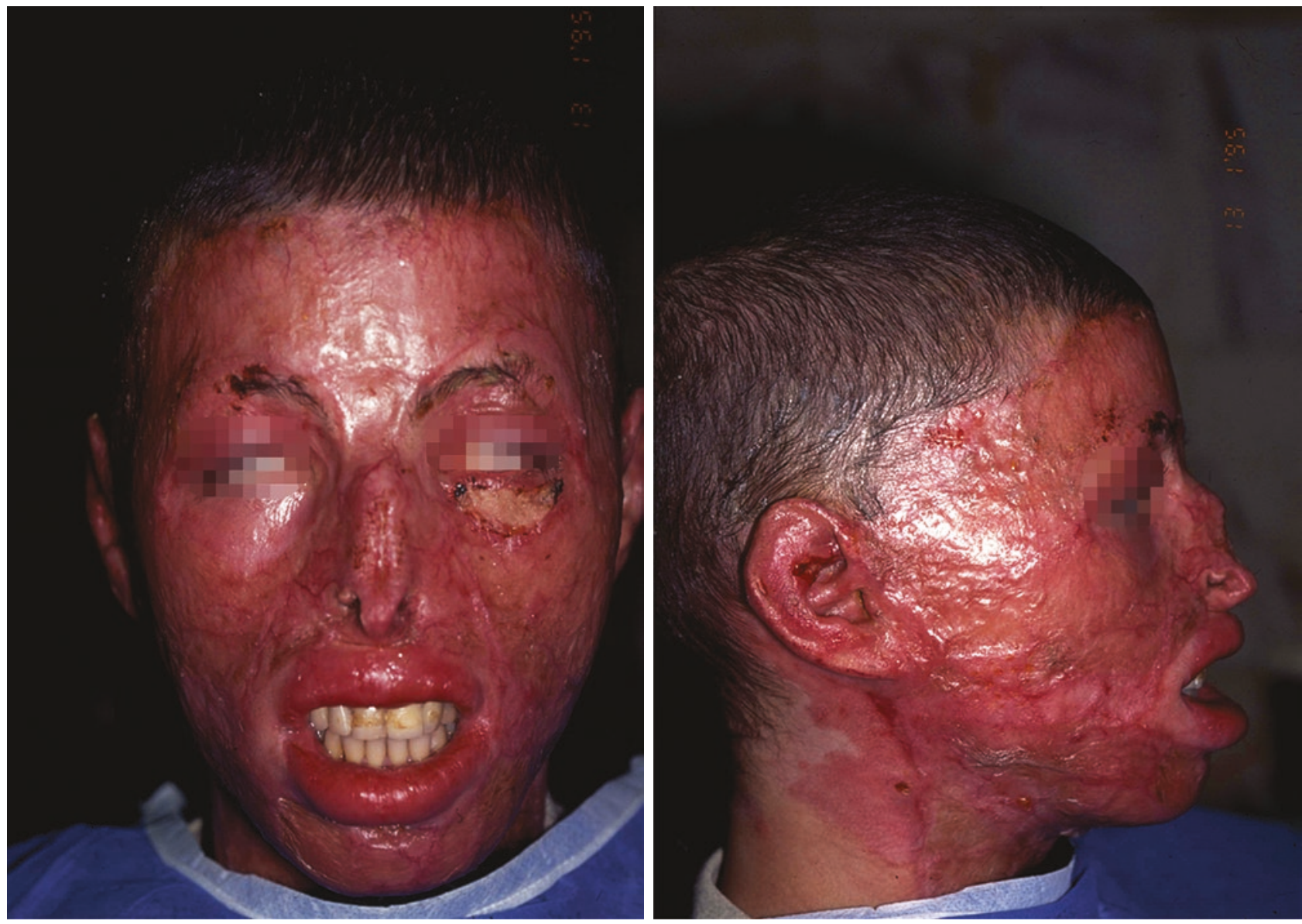

- Fig. 38.5 Preoperative aspect face and profile female 28 years old

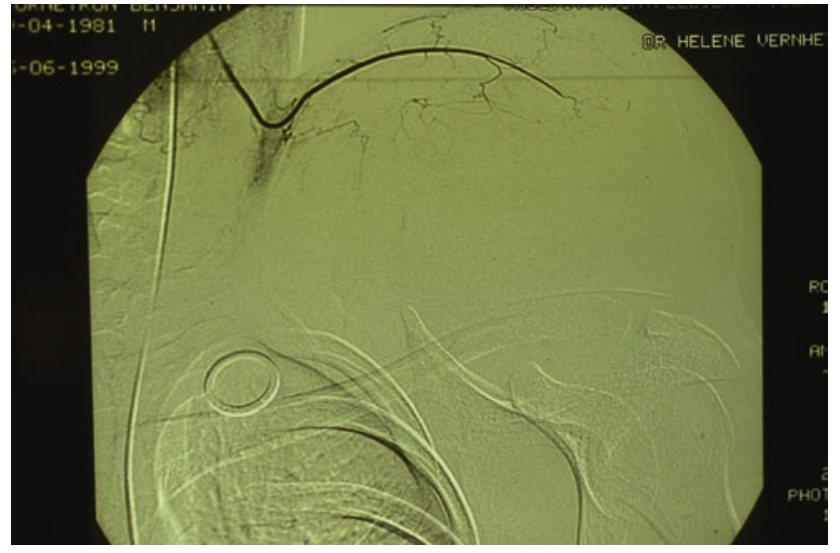

- Fig. 38.6 Arteriogram before the second procedure. Note the good vascularization of the pedicle and the branches covering the freshly expanded skin area

life and social exclusion due to scarring as a medical consideration comparable to a life-threatening situation, as it imposed an immunosuppression for life.

In 2015, 12 partial and 5 full facial transplants were recorded in the literature. Procedures included partial and near-total facial myocutaneous flaps and complex osteomyocutaneous grafts [5]. Fifteen patients received fully vascularized grafts, and 2 patients died of transplant-related and infectious complications

Facial transplantation was proposed to restore quality of life and enable social reintegration. Results publishing the first facial transplants created some polemics all over the world. The main discussions were concerning the long-term aesthetic and functional outcomes where more precisely defined outcomes are expected. In addition, significant technical, medical, and ethical issues remain to be solved, such as the secondary late failure and need for a second transplantation, recently reported by two authors [6, 7].

In 2018, Ozkan et al. [8] presented their long-term experiences with a series of five face-transplanted patients in terms of surgical aspects and postoperative outcomes. Possible salvage strategies in case of difficulties were also described. Five patients, four receiving full-face transplantation and one undergoing partial transplantation were included. The 


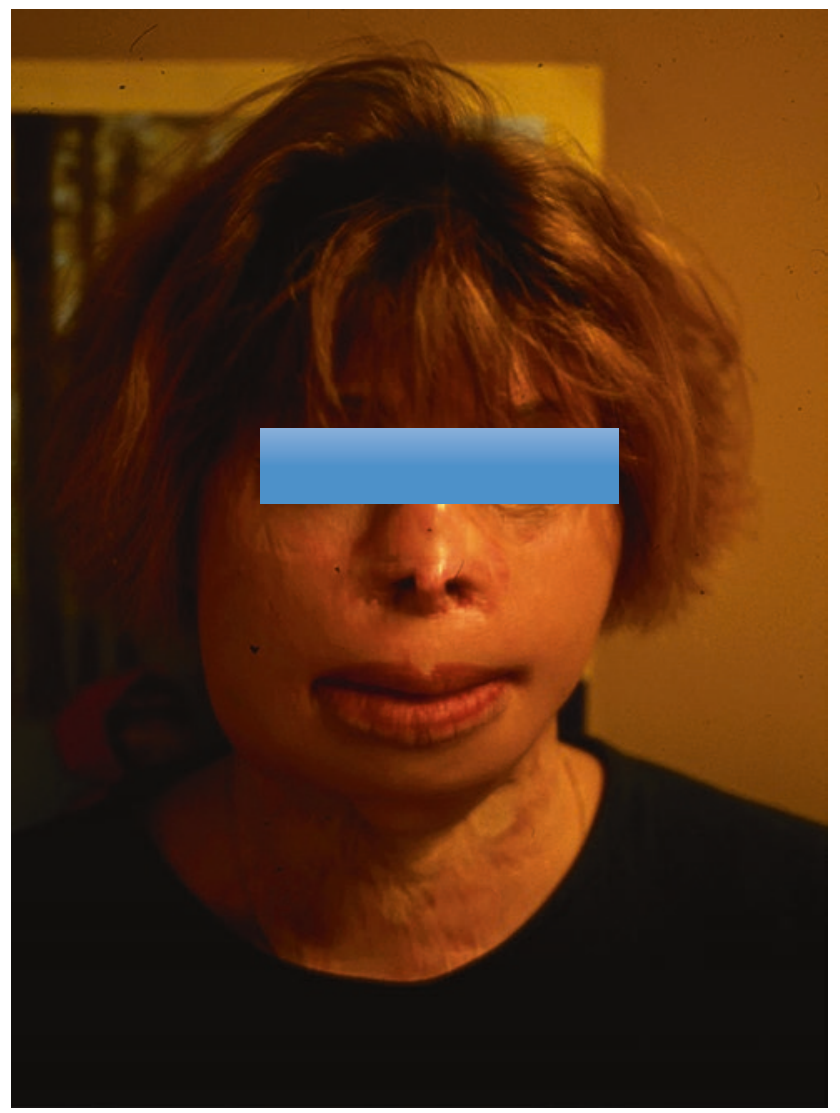

- Fig. 38.7 Four years postoperative, the quality of life has strongly improved. This young woman married, had a child, and returned to work

patients were aged between 19 and 54 years. Two had extensive burn scars to the face, and three had suffered gunshot injuries. The posttransplant induction immunosuppressive regimen included ATG plus tacrolimus, mycophenolate mofetil, and prednisone.

Patient files included their etiologies, preoperative preparations, surgical techniques, immunosuppressive regimen, postoperative courses, revisional surgeries, together with challenges including acute rejection episodes, and immunosuppressive drug complications.

No re-surgery due to vascular compromise was required in any case. One of the five patients was eventually lost due to complicated infectious and metabolic events 11 months posttransplantation. The other four patients were still alive, with a mean follow-up time of 53 months, and had satisfactory functional transplants and cosmetic appearance.

Potential complications may be linked to compliance and psychological maturity of the patients, the risk of opportunistic infections, and malignancies.
These situations need be resolved for it to be accepted as a safe procedure.

Advantages and Limits of the Homologous Transplantation

After more than 50 transplants, the concept of "face transplantation" has emerged even if several considerations should be mentioned: the immunological science used for these transplantations is derived from the ones used in organ transplantation, and the antirejection regimen should be adapted to the rejection levels of the transferred tissues, their rejection capacities depending on their structure and vascularization (skin, muscle, aponeurosis), etc. The number of cases already realized all over the world is limited, and each case has his/her own multifactorial problems, linked to their own medical history, the preoperative difficulties during transplantation, the rate of opportunistic infections, and the compliance of the patient [9].

The ethical discussions ended with a mandatory preliminary authorization for transplantation in most of the countries where they were realized. This frame has limited the number of cases but opened a disruption in the "no-harm" principle, due to the risk of potential complications induced by immunosuppression in a non-vital medical situation. However, the intense need for more social inclusion of these abandoned patients has transformed the conservative approaches into more progressive possibilities.

\subsection{Critical Analysis of the Literature}

The scarce literature concerning large face reconstruction is the reflection of the limited number of patients presenting a scar incompatible with a minimal social life or the difficulty to find surgical teams able to sustain a complex program including microsurgery, multiple actors, and adapted rehabilitation and care. The choice between the two strategies may be led by other considerations, like the ethical problem of submitting the patient to immunosuppression for the rest of his/ her life. Several authors have considered this as a progress opening the way to a large diffusion; others have simply refused to consider that facial tissues would be compared to any organ transplant performed on a daily basis all over the world like the kidney, pancreas, lung, or heart.

In conclusion, extensive facial scars remain a difficult challenge for reconstruction. Even in highly sophisticated teams, the capacity of a homologous facial transplantation needs to be prepared. The technique demands 
a permanent immunosuppression, and even if the ethical problem was more or less solved, the opportunistic infections remain a risk, and the long-term consequences are unknown in terms of life-threatening issues. The techniques imposing a long-term preparation like skin expansion plus or minus microsurgical revascularization are more usual. The specificity of the transferred tissues is less fine than with a complete homologous transfer and will need complementary techniques (fatty tissue removal or fat injections, makeup, etc.). A more EBM-based approach is needed to compare the results and mainly quality of life after allotransplantation versus auto transfers.

\section{Take Home Message \\ Face disfiguration represent difficult cases where the technical capacities of restoring a normal face using long and complex surgical techniques have to be weighed to the motivation and understanding of the patient. Psychological testing should be realized before proposing surgery, in order to prevent disil- lusions and bad feelings afterward and to drive the patient toward the right choice to do. All potential complications have to be explained, as well as the length and risks of each procedure, keeping in mind that some strategies will need several procedures and that the final result will only be visible a long time after the last procedure.}

\section{References}

1. Nuri T, Ueda K, Fujimori Y. Ten-year follow-up after treating extended burn scar contracture with an autologous cultured dermal substitute. Plast Reconstr Surg Glob Open. 2018;6(6):e1782. https://doi.org/10.1097/GOX.0000000000001782.

2. Ma X, Li Y, Li W, Liu C, Liu H, Xue P, Cui J. Reconstruction of facial-cervical scars with pedicled expanded deltopectoral flap. J Craniofac Surg. 2017;28(6):1554-8. https://doi.org/10.1097/ SCS.0000000000003901.

3. Teot L, Cherenfant E, Otman S, Giovannini UM. Prefabricated vascularised supraclavicular flaps for face resurfacing after postburns scarring. Lancet. 2000;355(9216):1695-6.

4. Gao JH, Ogawa R, Hyakusoku H, Lu F, Hu ZQ, Jiang P, Yang L, Feng C. Reconstruction of the face and neck scar contractures using staged transfer of expanded "Super-thin flaps". Burns. 2007;33(6):760-3.

5. Garrett GL, Beegun I, D'souza A. Facial transplantation: historical developments and future directions. J Laryngol Otol. 2015;129(3):206-11. https://doi.org/10.1017/ S0022215114003478

6. Morelon E, Petruzzo P, Kanitakis J, Dakpé S, Thaunat O, Dubois V, Choukroun G, Testelin S, Dubernard JM, Badet L, Devauchelle B. Face transplantation: partial graft loss of the first case 10 years later. Am J Transplant. 2017;17(7):1935-40. https://doi.org/10.1111/ajt.14218.

7. Lantieri L: report of a facial transplantation failure and need for second procedure. Dublin 2018, Make Better Summit.

8. Özkan Ö, Özkan Ö, Ubur M, Hadimioğlu N, Cengiz M, Afşar İ. Face allotransplantation for various types of facial disfigurements: a series of five cases. Microsurgery. 2018;38(8):834-43. https://doi.org/10.1002/micr.30272.

9. Giatsidis G, Sinha I, Pomahac B. Reflections on a decade of face transplantation. Ann Surg. 2017;265(4):841-6. https://doi. org/10.1097/SLA.0000000000001760.

Open Access This chapter is licensed under the terms of the Creative Commons Attribution 4.0 International License (http://creativecommons. org/licenses/by/4.0/), which permits use, sharing, adaptation, distribution and reproduction in any medium or format, as long as you give appropriate credit to the original author(s) and the source, provide a link to the Creative Commons license and indicate if changes were made.

The images or other third party material in this chapter are included in the chapter's Creative Commons license, unless indicated otherwise in a credit line to the material. If material is not included in the chapter's Creative Commons license and your intended use is not permitted by statutory regulation or exceeds the permitted use, you will need to obtain permission directly from the copyright holder. 